\title{
Optical sensors based on lossy-mode resonances
}

\author{
Ignacio Del Villar, ${ }^{1 *}$ Francisco J. Arregui, ${ }^{1,2}$ Carlos R. Zamarreño, ${ }^{1}$ Jesus M. Corres, ${ }^{1}$
} Candido Bariain, ${ }^{2}$ Javier Goicoechea, ${ }^{2}$ Cesar Elosua, ${ }^{2}$ Miguel Hernaez, ${ }^{2}$ Pedro J. Rivero, ${ }^{2}$ Abian B. Socorro, ${ }^{1}$ Aitor Urrutia, ${ }^{2}$ Pedro Sanchez, ${ }^{2}$ Pablo Zubiate, ${ }^{2}$ Diego Lopez, ${ }^{2}$ Nerea De Acha, ${ }^{2}$ Joaquin Ascorbe, ${ }^{2}$ Ignacio R. Matias ${ }^{1}$

${ }^{1}$ Institute of Smart Cities (ISC), Public University of Navarra, 31006 Pamplona, Spain

${ }^{2}$ Electrical and Electronic Engineering Department, Public University of Navarra, 31006 Pamplona, Spain.

* Corresponding author: ignacio.delvillar@ unavarra.es

\begin{abstract}
Lossy-mode resonance (LMR)-based optical sensing technology has emerged in the last two decades as a nanotechnological platform with very interesting and promising properties. LMR complements the metallic materials typically used in surface plasmon resonance (SPR)-based sensors, with metallic oxides and polymers. In addition, it enables one to tune the position of the resonance in the optical spectrum, to excite the resonance with both transverse electric (TE) and transverse magnetic (TM) polarized light, and to generate multiple resonances. The domains of application are numerous: as sensors for detection of refractive indices voltage, $\mathrm{pH}$, humidity, chemical species, and antigens, as well as biosensors. This review will discuss the bases of this relatively new technology and will show the main contributions that have permitted the optimization of its performance to the point that the question arises as to whether LMR-based optical sensors could become the sensing platform of the near future.
\end{abstract}

Keywords: optical sensor, resonance, thin-film, waveguide, hydrogel, biosensor

\section{INTRODUCTION}

In recent years, the deposition of thin films has permitted the development of numerous applications in important domains such as optical communications, optical microscopy, and photovoltaics [1,2]. In the field of sensor research, the development of the first surface plasmon polariton resonance-based sensor in 1982 was a scientific breakthrough [3]. It used the 
Kretschmann-Raether configuration [4] (see Fig. 1(a)), which basically consists of an optical prism on which a 56-nm-thick silver coating had been deposited. This setup permits surface plasmon polaritons to be generated at the metal-dielectric interfaces and to couple light at specific wavelength ranges. For this reason, this phenomenon is called surface plasmon polariton resonance or, for the sake of simplicity, surface plasmon resonance (SPR). The position of the resonance in the spectrum is very sensitive to the thin-film thickness and to the surrounding medium. If a layer sensitive to biological or chemical species is set on top of the metallic layer, then a biosensor or a chemical sensor is obtained [5]. In view of these interesting properties, the number of publications has increased exponentially, especially for detecting chemical and biological species [6,7], and there are some companies exploiting these devices especially for biosensing (Biacore http://www.biacore.com, Bionavis http://www.bionavis.com, and Xantec Bioanalytics http://www.xantec.com/).

Unfortunately, the sensitivity limit for SPR sensors seems to have been attained [8]. However, there is still another phenomenon that can be obtained with the Kretschmann configuration. In the same year as the first SPR sensor was developed, it was proved using a dielectric waveguide with a semiconductor waveguide clad that attenuation maxima in the transmission spectrum could be obtained for specific thickness values of cladding [9]. 


\section{(a) Setups for LMR and SPR generation}

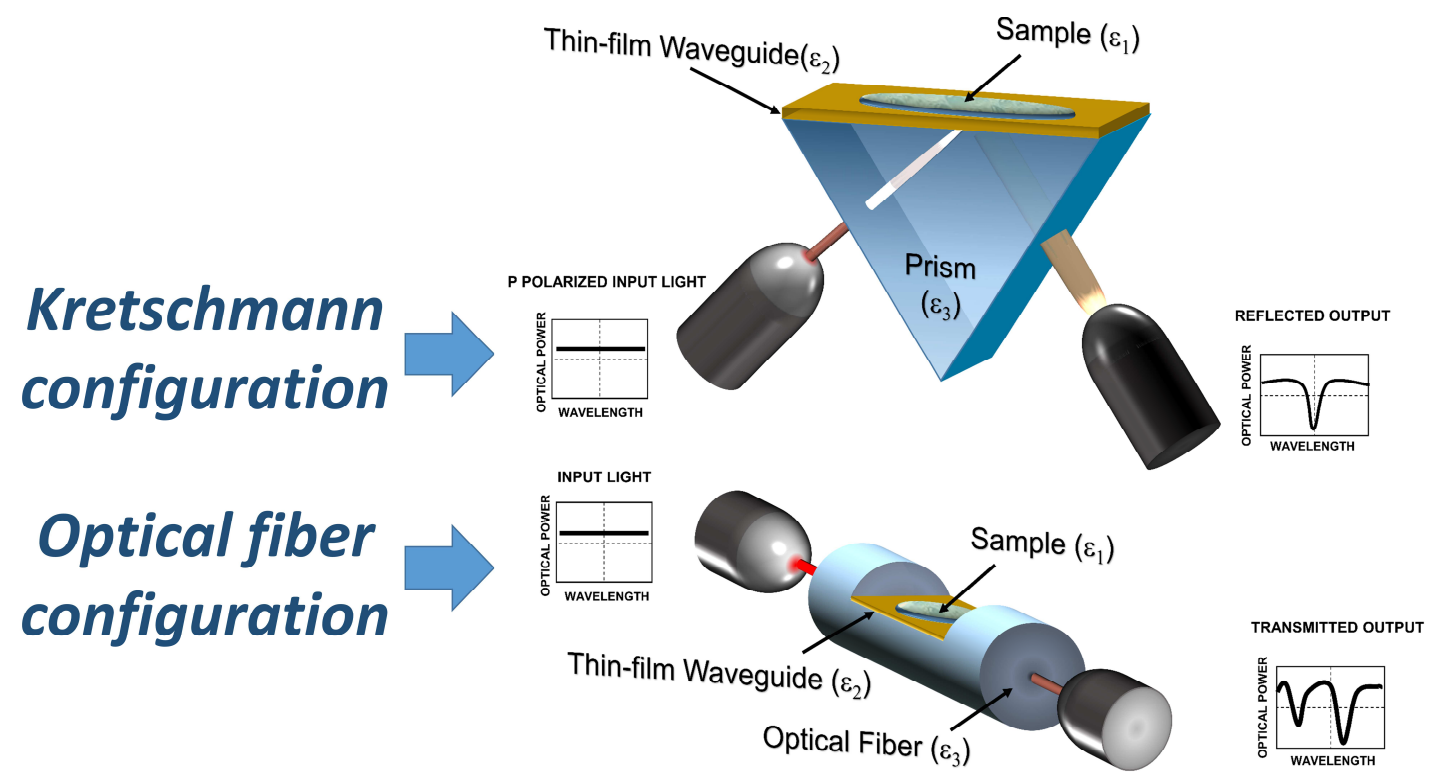

(b) LMR and SPR generation conditions

\begin{tabular}{|c|c|c|}
\hline TYPE OF RESONANCE & $\hat{\varepsilon}$ CONDITIONS & $\begin{array}{c}n, \boldsymbol{n} \text { CONDITIONS } \\
\forall \boldsymbol{n}>\mathbf{0}, \boldsymbol{k}>\mathbf{0}\end{array}$ \\
\hline $\begin{array}{c}\text { SPR } \\
\text { Surface Plasmon } \\
\text { Resonance }\end{array}$ & $\begin{array}{c}\mathcal{E}_{2}^{\prime}<0 \\
\left|\mathcal{E}_{2}^{\prime}\right|>\left|\mathcal{E}_{2}^{\prime \prime}\right| \\
\left|\mathcal{E}_{2}^{\prime}\right|>\left|\mathcal{E}_{3}^{\prime}\right|\end{array}$ & $k_{2}>(\sqrt{\mathbf{2}}+1) n_{2}$ \\
$k_{2}^{2}-n_{2}^{2}>1.45^{2}$
\end{tabular}

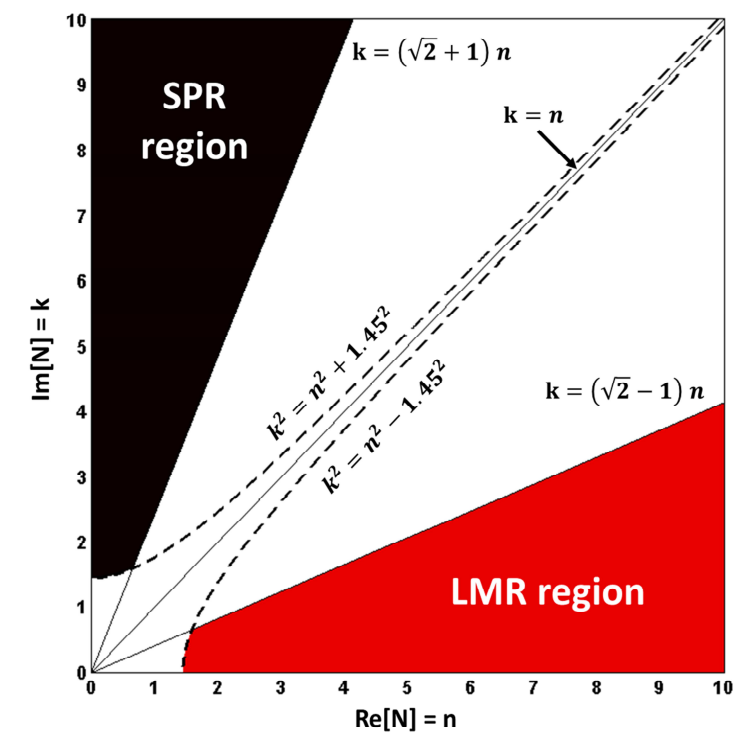

Figure 1. (a) Generation of LMR and SPR with a nanocoated optical prism (Kretschmann configuration) and a nanocoated D-shaped optical fiber. (b) Conditions for LMR and SPR generation in both configurations. 
On this basis it was proved later that it is possible to obtain a sensor for humidity, water, and alcohol vapor, or even n-heptane and iso-octane vapors, by using an anisotropic polymer deposited on a waveguide [10-12]. The basic principle of the measurements was to record the phase shift between the two index-matched modes after propagating through the waveguide.

However, the previous technique does not use a wavelength-based detection system, an idea explored in 1993 by Marciniack [13]. In that publication the lossy-mode resonances (LMRs) were observed with a wavelength sweep and explained as a coupling between dielectric waveguide modes and a lossy mode (a guided mode with a complex effective index) of the semiconductor-clad waveguide. Surprisingly, it was necessary to wait until 2005 to find a practical sensor configuration where LMRs are tracked as a function of wavelength [14]. Since that moment, a great number of works have been published, including the Kretschmann configuration setup [15,16] and a fiber-optic-based one [17-28] (see Fig. 1(a)), which has given rise to the necessity of presenting this topic to a broad scientific audience.

Waveguide modes can be classified as guided, leaky, and radiated [29]. Though some authors use the term guided-mode resonance for the phenomenon explained in this review [30], in view of the fact that a lossy mode is a specific type of guided mode, the term lossy-mode resonance has become popular in recent years [17,31-34]. That is why this term will be used henceforth. In this review, we will discuss the conditions for generation and optimization of the LMR sensors, and we will show the main applications developed during recent years.

\section{BASIC CONCEPTS IN LOSSY-MODE RESONANCES}

SPRs and LMRs can be observed in two similar structures: a thin-film-coated optical prism or a thin-film-coated optical fiber (see Fig. 1a). The optical spectrum position of the SPRs and LMRs depends on the external refractive index, and both phenomena can be used for the fabrication of highly sensitive refractometric sensors. In fact, the similarity in shape of LMRs and SPRs in the optical spectrum has led in some cases to false recognition of an SPR [35-37].

The first step in order to obtain an LMR is to choose an adequate material for the thin film. The conditions are very different from SPR generation. SPRs are obtained when the real part of the thin-film permittivity is negative and higher in magnitude than both its own imaginary part and the permittivity of the material surrounding the thin film, whereas LMRs occur when the real 
part of the thin-film permittivity is positive and higher in magnitude than both its own imaginary part and the material surrounding the thin film [30].

According to this, the expressions for SPR and LMR generation as function of permittivity $\left(\varepsilon=\varepsilon^{\prime}+i \varepsilon^{\prime \prime}\right)$ and refractive index (n is the real part and $\mathrm{k}$ the imaginary part) are presented in

Fig. 1(b) under the assumption of a substrate refractive index of 1.45 (with small variations, this is the value of silica in a broad range of the optical spectrum [38]) with a surrounding medium refractive index of 1 (air) [39].

Also in Fig. 1(b) a map is plotted containing the regions where LMRs and SPRs can be obtained. As long as the real part of the thin-film permittivity is positive and the absorption coefficient $(\mathrm{k})$ is low, many materials except pure metals (typical of SPRs) can induce LMRs. For instance, several contributions using metal oxides [17-23,40-46], polymer coatings [42,47-50], twolayer-coated structures combining both materials [22], or even immunosensors consisting of multilayers of polymers and antibodies [51,52] have successfully produced LMRs so far.

It is important to consider here that there are hybrid materials (e.g., indium tin oxide, ITO), which satisfy the conditions for LMR generation at certain wavelengths and the conditions for SPR generation at others $[15,17,18]$. Precisely with ITO, a material that permits the generation of both LMRs and SPRs, it has been observed that the optimum angle for exciting LMRs and SPRs differs (see Fig. 2(a)) [15]. This optimum angle is based on the influence of two parameters: refractive index and thickness. However, in the setup [15] the same thickness has been analyzed both for LMR generation at shorter wavelengths and for SPR generation at longer wavelengths. The result has been that the LMR is excited only at angles approaching $90^{\circ}$, whereas the SPR can be obtained at angles approaching $90^{\circ}$, but in a not optimal way. At $60^{\circ}$ a much better resonance is attained. In fact this last question agrees with other works where the best angle range for SPR excitation is $40-75^{\circ}[53,54]$, whereas for LMRs the optimum angles approach $90^{\circ}[55,56]$. Even though it is possible to move away from $90^{\circ}$ by modifying the refractive index (increasing the imaginary part) or changing the thickness, it is easier to obtain the LMR for angles approaching $90^{\circ}$ [55]. That is why the simplest and most effective structure for LMR generation consists of the optical fiber configuration, where the incident angles approach $90^{\circ}$ (see Fig. 1(a)).

As an example of optical fiber configuration, several transmission spectra from a claddingremoved multimode fiber coated with different materials are presented in Fig. 2(b). It is 
important to highlight that, contrary to surface plasmon polariton, LMRs are induced because a mode guided in the optical fiber experiences a transition to guidance in the thin film with losses. In Fig. 2(b), the real part of the effective index of mode $\mathrm{EH}_{1,9}$ starts to increase more than the rest of modes at wavelength $850 \mathrm{~nm}$, where the transmission through the optical fiber is lower (maximum LMR depth) until it exceeds the refractive index of the optical fiber (silica). This indicates that the mode is guided by the thin film of a higher refractive index than the silica substrate. The same occurs for $\mathrm{HE}_{1,11}$ mode, which induces the resonance located at $700 \mathrm{~nm}$ [57]. The coating thickness in all the spectra of Fig 2(b) is $200 \mathrm{~nm}$ and the real part of the refractive index is 1.55 , a typical value for polymeric coatings. Depending on the imaginary part of the refractive index, a clear dip can be obtained or not in the optical spectrum. For a purely real refractive index (imaginary part $=0$ ) of the thin film, there is a small perturbation in the spectrum due to interferometric effects (see plot a). This perturbation is caused by the modal effective index mismatch between the region where the thin film has been deposited (the middle region) and the region where the thin film has not been deposited (the input and output region). By increasing the imaginary part of the thin-film refractive index, two dips are induced in the optical spectrum (see plot b). This attenuation band is caused by the guidance of two lossy modes in the thin film, which causes an increase in the imaginary part of the effective index of modes guided in the substrate (see Fig. 2(b)). If the imaginary part of the thin-film refractive index is increased (plot c), the two dips overlap each other and it is only possible to distinguish one attenuation band. If the imaginary part is further increased (plot d), the effects of transmission losses overcome those of the resonance region, and the optical spectrum is flat. Therefore, the first conclusion is that an adequate imaginary part, neither too high nor too low, must be selected, which permits one to obtain a narrow resonance. 
(a) Optical prism
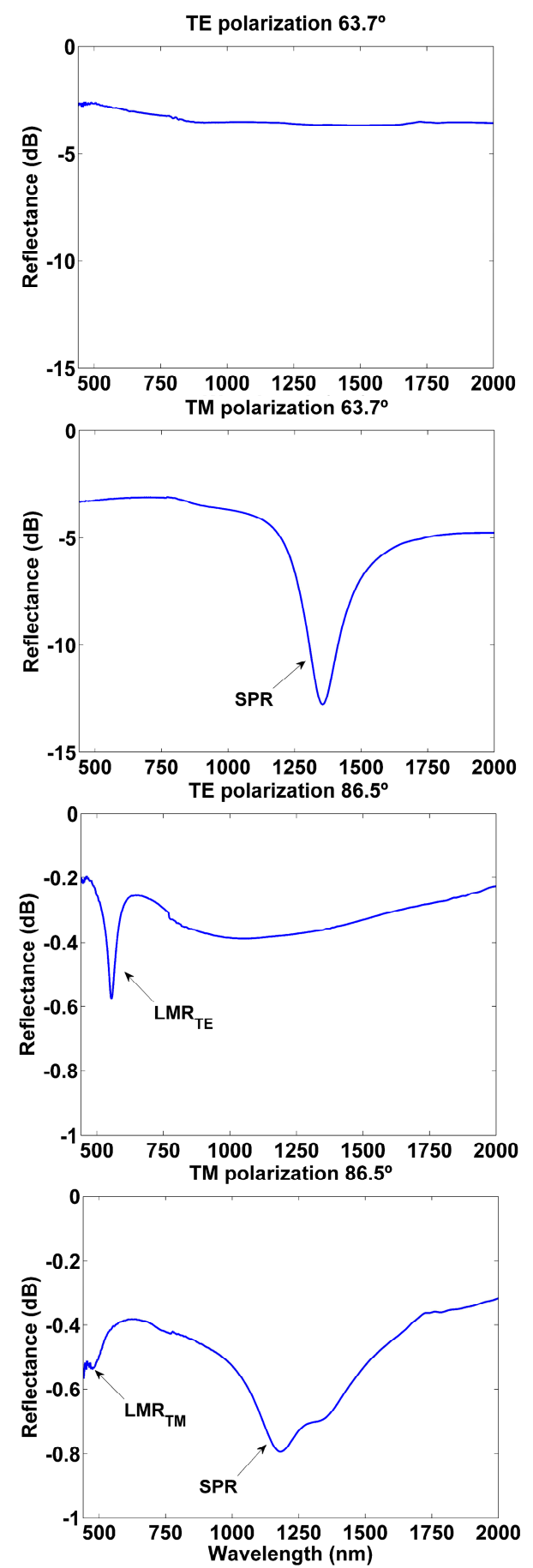

\section{(b) Optical fiber}
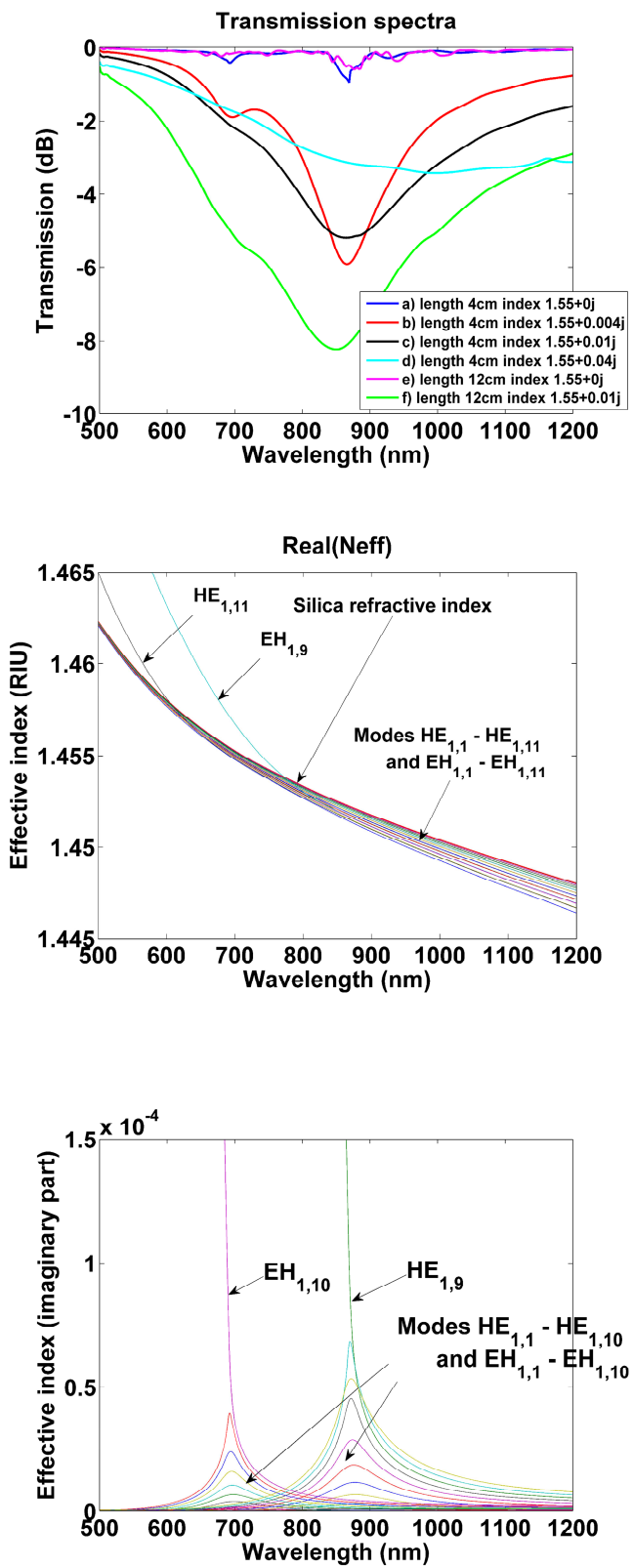

Fig. 2. (a) SPRs and LMRs obtained with the same Kretschmann configuration at different incidence angles $\left(63.7^{\circ}\right.$ and $\left.86.5^{\circ}\right)$ and polarizations (TE and TM). (b) Lossy-mode resonances obtained for different values in the imaginary part of the refractive index and different coating lengths. Figure reproduced with permission from: a, ref. [15]. 
It is also important to note that for a purely real refractive index, the increase in length leads to the generation of more fringes in the spectrum (plot e in Fig. 2(b)), confirming the interferometric nature of this phenomenon, whereas for a refractive index with an imaginary part 0.01 , the increase in length amplifies the depth of the resonance while maintaining the shape of the resonance (plot $\mathrm{f}$ ). This indicates that LMR it is not an interferometric phenomenon.

Besides the imaginary part of the thin-film refractive index, three other parameters must also be considered for a complete optimization of the sensor: the real part of the refractive index, the thin-film thickness, and the surrounding-medium refractive index (SRMI) [42]. In other words, LMR devices can be sensitive to the refractive index and thickness of the thin film and to the surrounding-medium refractive index as well. This makes possible multiple configurations for sensing: a thin film that changes its thickness (swells) depending on a parameter, a thin film that changes its refractive index depending on the target, or a refractometric device that is sensitive to the surrounding refractive index. Moreover, it is possible to deposit a thin film for the generation of LMRs and a second overlay that acts as the sensitive coating [22,25].

As a general rule, the thin-film refractive index and thickness, and SRMI must be increased in order to obtain a higher sensitivity [42]. However, there is a limitation. The detection method is based on tracking the central wavelength of the LMR. Since the LMR peak is progressively redshifted as a function of the refractive index and the thickness of the thin film, there is a moment when the LMR can no longer be monitored due to the limited spectral range of the spectrum analyzer. The solution is to follow the second LMR, which is obtained when a second mode is guided in the thin film, then the third LMR, which corresponds to the third mode guided in the thin film, and so on. This strategy would broaden the measurement range, but, as a counterpart, the second mode transition is less abrupt than the first one (less sensitive), the third transition is less abrupt than the second one, and so on. As an example, the transmitted power as a function of coating thickness and wavelength is shown in Fig. 3 for materials with different refractive indices: $\mathrm{TiO}_{2} / \mathrm{PSS}$ and PAH/PAA, with 1.92 to 2.11 and 1.5 to 1.47 , respectively, in the wavelength range analyzed. In both cases, the sensitivity (wavelength shift vs increase in thickness) is higher for the first LMR. However, if the first LMR obtained with both materials is compared, the sensitivity with $\mathrm{TiO}_{2} / \mathrm{PSS}$ is higher. Consequently, the basic rule is to track the first LMR for the highest real part of the refractive index. The thickness can be used to set the LMR at the desired wavelength position. In video S1 the experimental and theoretical evolution 
of LMRs in the PAH/PAA-coated cladding removed multimode fiber (CRMF) as a function of coating thickness is shown.

Another rule [42] is to increase the SMRI, but the former parameter is usually fixed by the application (e.g., in biological or chemical applications, the medium is water: 1.33). Consequently, the key parameter is the real part of the refractive index thin film.

\section{REDUCING THE SPECTRAL WIDTH OF LOSSY-MODE RESONANCES}

As was been observed in the previous section, the adequate selection of the imaginary part of the thin-film refractive index permits one to reduce the spectral width of the LMR (see Fig. 2). However, by following the rule of tracking the first LMR for a higher sensitivity, this LMR presents a higher spectral width than the rest of the LMRs [58]. As an example, for the PAH/PAA-coated CRMF (see Fig. 3), the first LMR obtained with a thickness of $750 \mathrm{~nm}$ presented a higher spectral width than the second LMR obtained with a thickness of $1200 \mathrm{~nm}$. Consequently, the figure of merit, expressed as the ratio between the sensitivity to surrounding refractive index (SRI) and the full-width at half maximum (FWHM), was only 8 [58]. This value is one-tenth of that obtained with SPRs [59]. 


\section{(a) $\mathrm{TiO}_{2} / \mathrm{PSS}$}
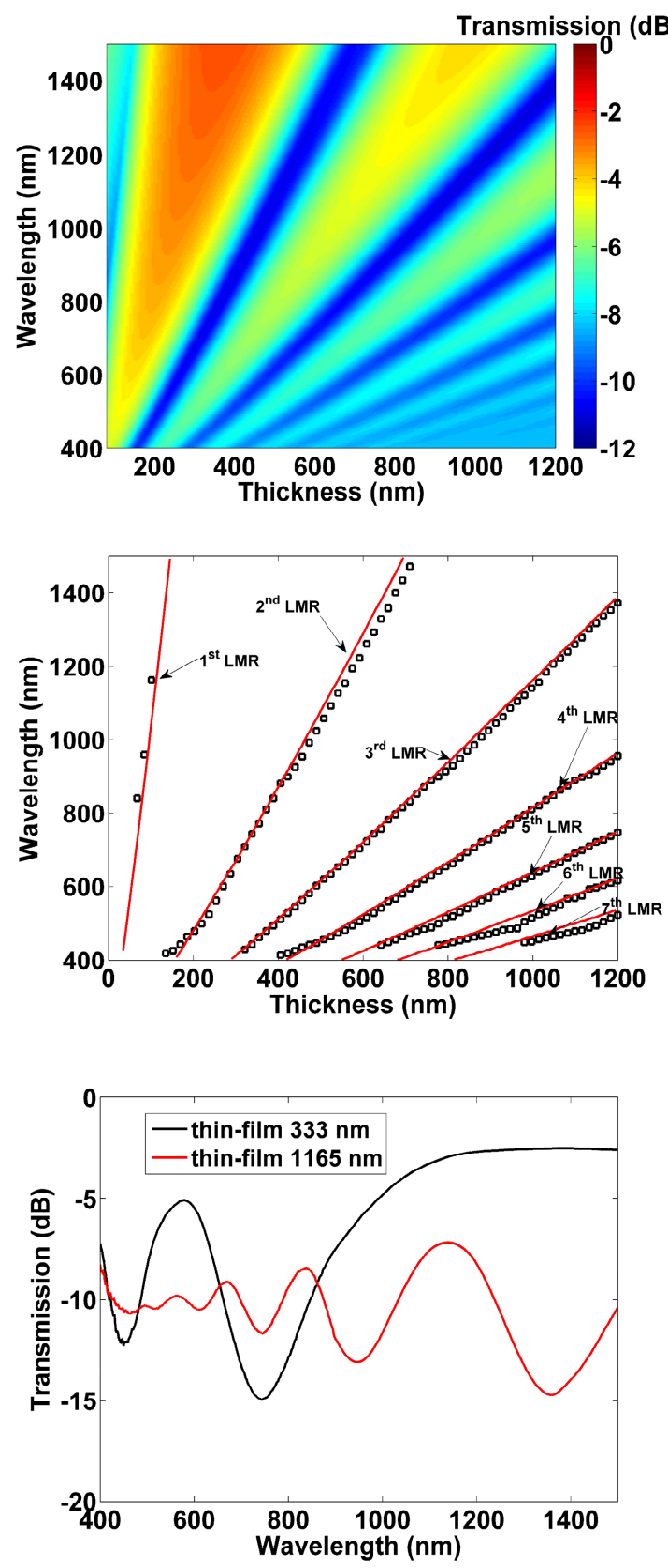

(b) PAH/PAA
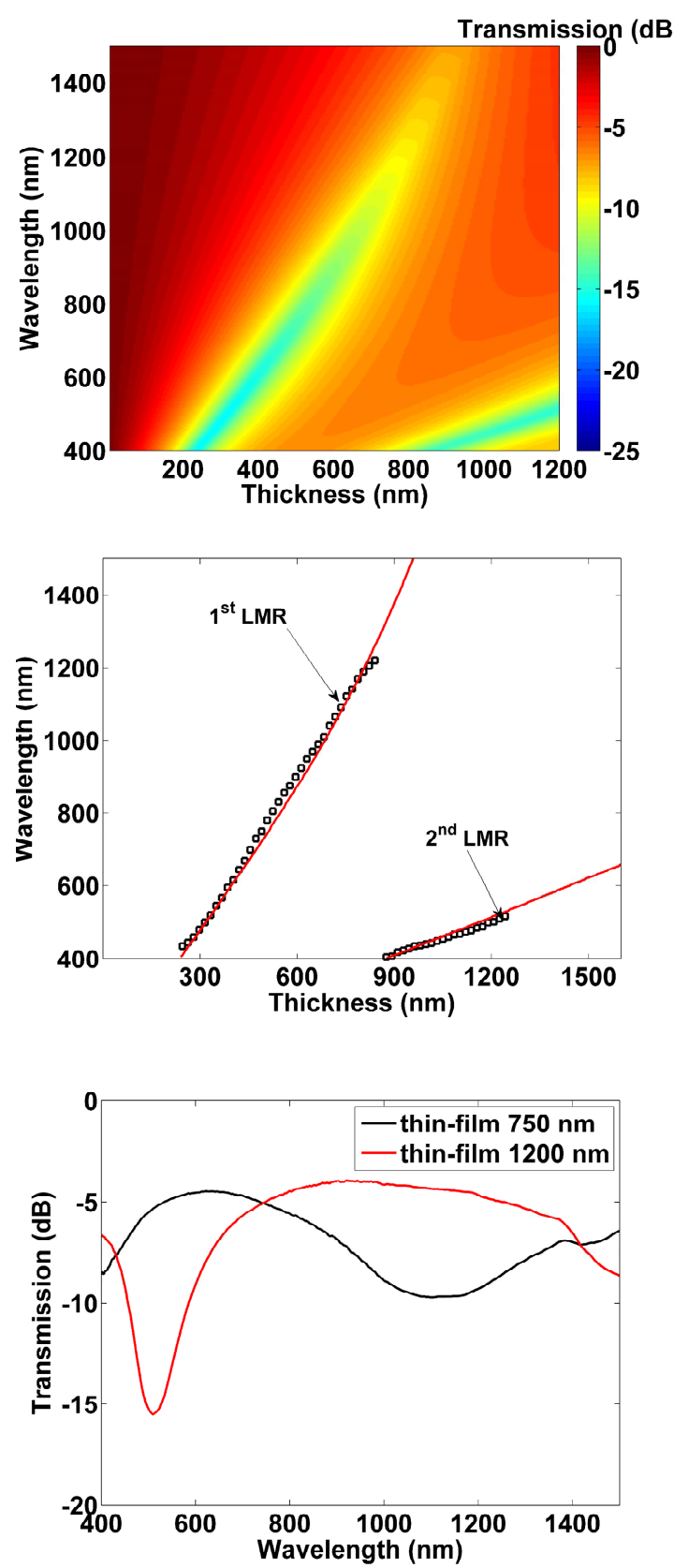

Fig. 3. Spectral sweep as a function of coating thickness, resonance wavelengths (numerical data are shown by a solid line and experimental data by squares) as a function of coating thickness, and two spectra obtained for two coating thickness values. Materials analyzed: (a) $\mathrm{TiO}_{2} / \mathrm{PSS}$ (refractive index: 1.92-2.11) and (b) PAH/PAA (refractive index: 1.47-1.5). Figure reproduced with permission from ref. [42], () 2012 OSA. In video S1 the experimental and theoretical evolution of LMRs in the PAH/PAA-coated cladding removed multimode fiber (CRMF) as a function of coating thickness is shown. 
In order to solve this issue, optimized optical fiber waveguides can be used: adiabatic tapered single-mode fiber (ATSMF) [52] and D-shaped fiber [44]. Regarding the ATSMF structure, it is important to indicate that light is transmitted in the waist of the tapered fiber through a single mode, whereas in a CRMF structure, light is transmitted through multiple modes. Consequently, LMRs in a CRMF structure are due to the attenuation of each of the substrate modes (modes guided through the optical fiber) when one of these modes starts to be guided in the thin film [55]. The maximum attenuation of each of the modes guided in the substrate occurs at different wavelengths and, as a result, the spectral width is higher than in an ATSMF structure, where a single coupling takes place. As a result, a two-fold spectral width reduction can be obtained [52] (see Fig. 4(a)).

A second way of reducing the spectral width is by using a D-shaped fiber, which also shows a single coupling of light from the core of the optical fiber to the thin film. This structure has an additional advantage, which is related to the guidance of modes in the thin film in pairs.

For thin films with a refractive index that does not exceed the substrate refractive index by a large amount (see Fig. 4(c)), each pair of modes is guided in the thin film at a similar wavelength. Hence, the LMR obtained is actually a combination of two different resonances that correspond to the two types of lossy modes guided in the thin film.

Most studies have been based on CRMF structures, where the cross-section shows a cylindrical geometry $[17,19,42,47]$. Consequently, the two types of modes guided are hybrid (HE and EH) modes. On the other hand, the D-shape fiber uses a thin film whose cross-section is rectangular [44]. As a result, the modes guided in the thin film can be either transverse electric (TE) or transverse magnetic (TM). In order to avoid confusion, the two LMRs will be considered in all cases as $\mathrm{LMR}_{\mathrm{TE}}$ and $\mathrm{LMR}_{\mathrm{TM}}$.

The superposition of both resonances leads to a spectral width increase, which can be avoided by increasing the refractive index of the thin film, which permits one to guide the modes at quite separated wavelengths and to distinguish $\mathrm{LMR}_{\mathrm{TE}}$ from $\mathrm{LMR}_{\mathrm{TM}}$ (see Fig. 4(d)). 


\section{(a) CRMF vs tapered SMF (b) CRMF vs D-shaped fiber}

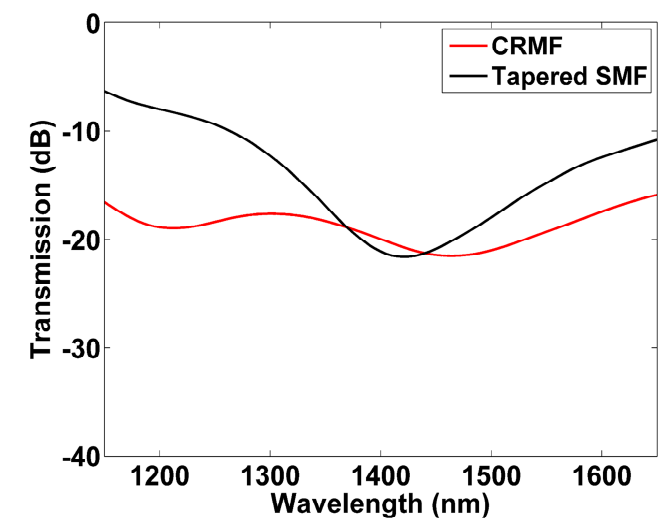

(c) ITO coated CRMF

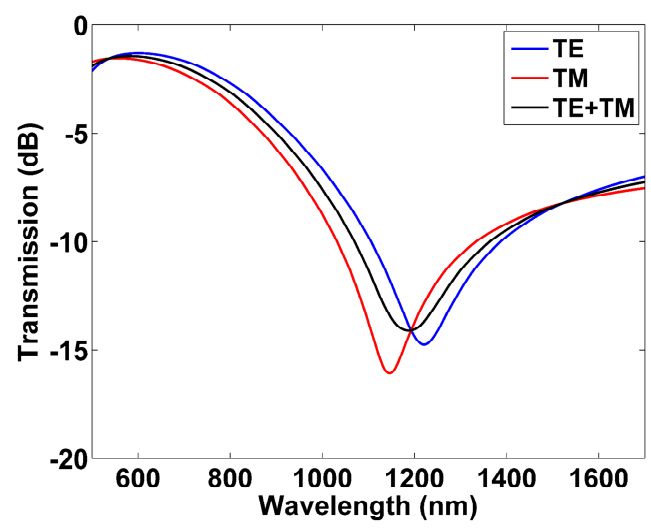

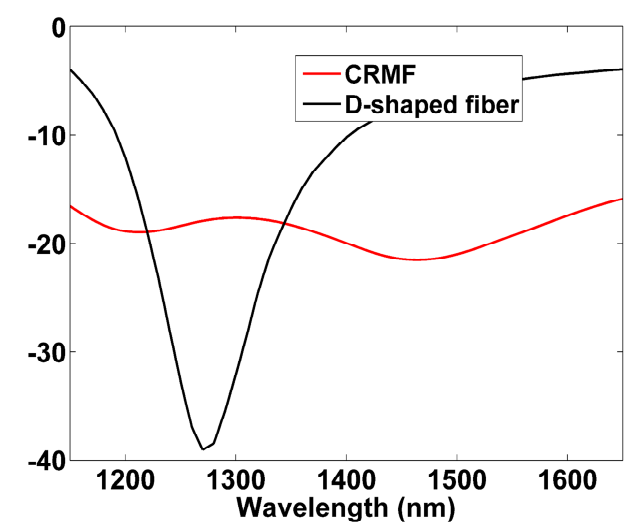

(d) $\ln _{2} \mathrm{O}_{3}$ coated CRMF

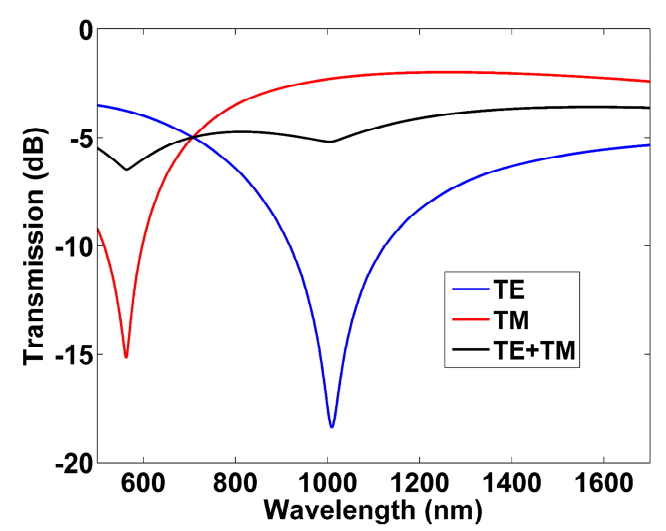

Fig. 4. (a) Comparison of LMRs obtained with a CRMF structure and a tapered single-mode fiber. (b) Comparison of LMRs obtained with a CRMF structure and a D-shaped fiber. (c) ITO-coated LMR in a CRMF structure (TE and TM LMRs overlap and only one attenuation band is obtained). (d) Indium oxide-coated LMR in a CRMF structure (TE and TM LMRs do not overlap and two attenuation bands are obtained). Figure reproduced with permission from: a,b ref. [60], @ 2015 IEEE; c, d ref. [18], @ 2010 IOP.

D-shaped fiber can help to solve this uncertainty. Due to its nonuniform azimuthal index variation, the spectral transmission for TE or TM polarized light is different [44-46]. Consequently, it is possible to separate the $\mathrm{LMR}_{\mathrm{TE}}$ and $\mathrm{LMR}_{\mathrm{TM}}$ bands by exciting the optical fiber with TE or TM polarized light. Thanks to this property, the spectral width of a D-shaped fiber is even lower than that of an ATSMF. In Fig. 4(b), a fourfold improvement is observed if we compare the $30 \mathrm{~nm}$ spectral width of the D-shaped fiber and the $125 \mathrm{~nm}$ spectral width of the ATSMF. 


\section{REFRACTIVE INDEX-SENSING APPLICATIONS}

The LMR devices with the highest number of publications is refractometers [14,17$20,23,26,27,40-42,61,62]$. This is because the refractive index response is a simple indicator of how sensitive the device will be to chemical and biological species [6], which is one of the most important research fields of optical fiber sensors nowadays.

Metallic oxides and polymers are among the materials that permit the generation of LMRs. The first group is more adequate than the second because the material refractive index is typically higher and, as was indicated above, a higher refractive index in the thin film leads to a higher sensitivity [42]. Even so, it is surprising that there are no experimental works on LMRs based refractometers with a polymer coating. The reason is that refractometers are typically tested with oil or glycerol solutions, which adhere to the rough surface of the polymer, which prevents an adequate characterization. On the other hand, metallic oxides present a much flatter surface, which permits the development of many LMR refractometers based on ITO [17,18,20,23,26,40,41], indium oxide [18,21], $\mathrm{TiO}_{2} / \mathrm{PSS}$ [19,42], and $\mathrm{SnO}_{2}$ [61] coatings. Among these, ITO is the only material that enables the generation of LMRs and SPRs at shorter and higher wavelengths, respectively. Due to ITO's special characteristics, the general rule of increasing the thickness for a higher sensitivity is not applicable when the LMR band approaches the SPR region. In this case, the LMR wavelength shift as a function of the thickness is progressively reduced until it reaches the limit wavelength of the SPR region. This explains why the first LMR exhibits a sensitivity of $2952.6 \mathrm{~nm} / \mathrm{RIU}$ with a $115 \mathrm{~nm}$ coating, whereas a lower sensitivity of $1617.4 \mathrm{~nm} / \mathrm{RIU}$ is attained with a $220 \mathrm{~nm}$ coating [17].

The limit wavelength, which depends on the refractive index dispersion, can be controlled with the thin-film fabrication conditions [56]. For instance, with DC sputtering and a post-annealing it has been possible to obtain ITO-coated LMR-based sensors with a limit wavelength of $800 \mathrm{~nm}$ [40], whereas with dip coating technique this value is shifted to $1400 \mathrm{~nm}$ [17].

The rest of the materials satisfy the conditions for LMR generation in the entire optical spectrum (typically 400-1500 nm) and follow the rules indicated by Del Villar [42]. Consequently, the sensitivity increases linearly as a function of the thickness. $\mathrm{TiO}_{2}$, with a refractive index of 1.971.74 in the wavelength range $400-1700 \mathrm{~nm}$, permits one to obtain a sensitivity of $2872 \mathrm{~nm} / \mathrm{RIU}$ in the refractive index range of 1.321 (water) to 1.43 (close to silica index) [19]. Indium oxide, with a refractive index of 2.09-1.84 in the wavelength range $400-1700 \mathrm{~nm}$, permits one to obtain 
a sensitivity of $4068 \mathrm{~nm} / \mathrm{RIU}[18,21]$. $\mathrm{SnO}_{2}$, with higher refractive index than $\mathrm{TiO}_{2}$, and indium oxide, permits one to obtain a sensitivity of $5390 \mathrm{~nm} / \mathrm{RIU}$ [61]. Moreover, with $\mathrm{Al}_{2} \mathrm{O}_{3}$ and a ATSMF structure it has been possible to attain $6008 \mathrm{~nm} / \mathrm{RIU}$ [63].

As an example of the performance of refractometers, in Fig. 5(a) the central wavelength of the LMR in a $\mathrm{SnO}_{2}$-coated CRMF is progressively shifted to the red as a function of the refractive index. The device can also be used as a visible light optical filter, and the refractive index can be interpreted by the color of the output light (see the photograph in Fig. 5(a)).

In Fig. 5(b), another refractometer with a $\mathrm{TiO}_{2}$ coating is shown. Several resonances can be tracked with increasing sensitivity for lower-order LMRs [19].
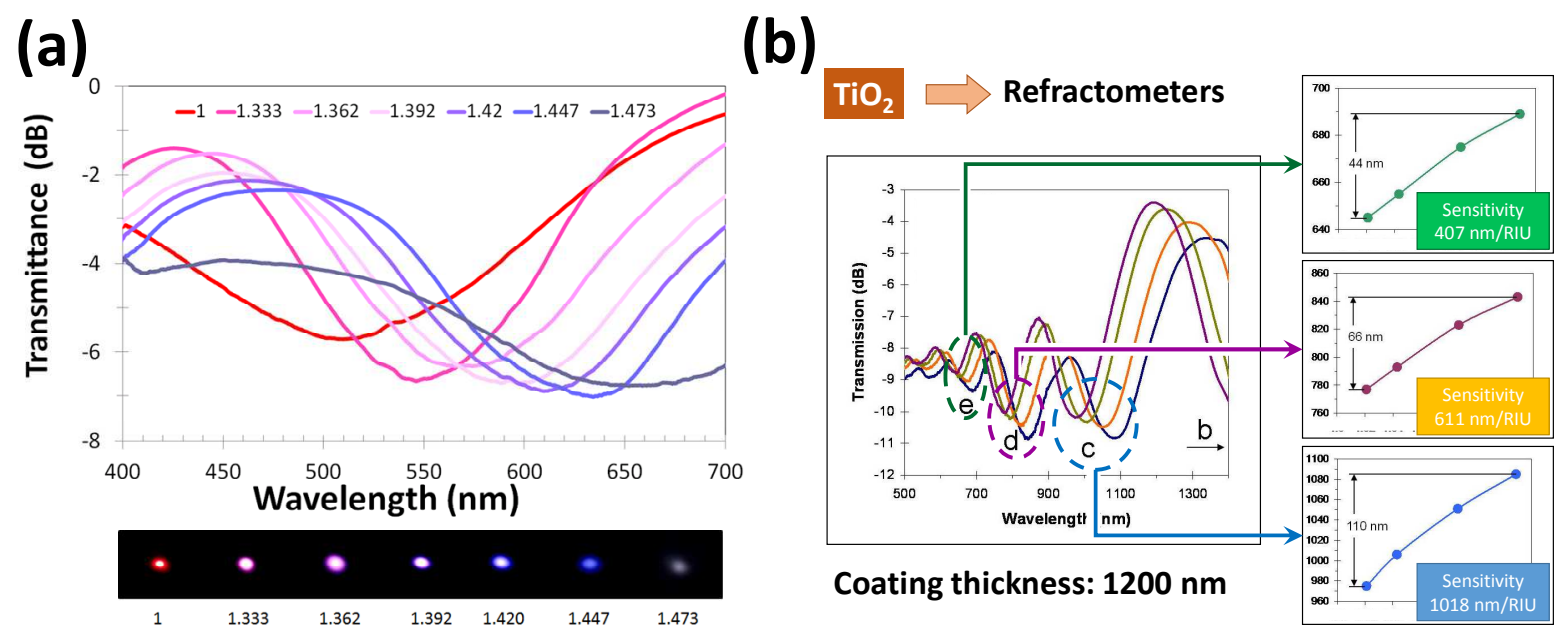

Fig. 5. (a) Coated CRMF-based sensor with a $110 \mathrm{~nm} \mathrm{SnO}$ coating. The LMR shifts to the red as a function of the external refractive

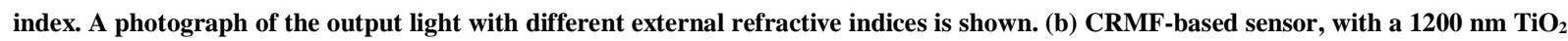
coating. The thicker coating permits one to obtain several LMRs with different sensitivities. Figure reproduced with permission from: b, ref. [19], (C) 2010 OSA.

The previous refractometers were fabricated with a single coating. However, there are some theoretical works where two coatings are used with the aim of optimizing the sensitivity. A first possibility is the combination of ITO and $\mathrm{TiO}_{2}$ [32], which permits a twofold increase in sensitivity compared to the results obtained with a single ITO layer. Another promising idea is to include a low refractive index matching layer in between the substrate and an ITO coating. This permits one to obtain a sensitivity of $4652 \mathrm{~nm} / \mathrm{RIU}$, a fourfold increase compared to the same device without the index matching layer [33], and resembles the setup used for enhancing the sensitivity in SPR-based sensors [64]. Consequently, the path toward multilayer structures has 
been opened, and this could lead to an enhancement of the performance and the quality of LMRbased sensors.

In the meantime, even with a single coating, it has been possible to attain record sensitivities with the D-shaped fiber configuration. This is possible because the sharp resonances obtained with this structure can be tracked at long wavelengths, where the sensitivity is higher. With a silicon coating, a sensitivity of $5700 \mathrm{~nm} / \mathrm{RIU}$ in the range of 1.33-1.38 was obtained [14], whereas with a $\mathrm{ZnO}$ coating, a sensitivity of 3000-6700 nm/RIU in the range of 1.33-1.4 was attained [27]. With ITO it has been possible to obtain $6009 \mathrm{~nm} / \mathrm{RIU}$ in the refractive index range of $1.365-1.38$ [44] and $19351 \mathrm{~nm} / \mathrm{RIU}$ in the 1.415-1.423 region [65].

Finally, according to the rules indicated by Del Villar [42], a material with higher refractive index than ITO has been deposited: $\mathrm{SnO}_{2}$. This permitted one to obtain a sensitivity of 14510 $\mathrm{nm} / \mathrm{RIU}$ in the water region (refractive index: 1.33) [66], a value that approaches the best optical sensors (i.e., long-period fiber gratings, where $20000 \mathrm{~nm} / \mathrm{RIU}$ has been obtained in the water region [67], and SPR sensors, with $57000 \mathrm{~nm} / \mathrm{RIU}$ obtained in water [64]). However, the most interesting point is that also with the D-shaped it has been possible to attain $304360 \mathrm{~nm} / \mathrm{RIU}$ in the silica refractive index region [66]. With reversed symmetric waveguides, where the substrate material is changed [68], this value could be obtained in the water region. The only requirement is to use an optical fiber with a refractive index similar to water.

\section{ENVIRONMENTAL PARAMETERS AND CHEMICAL AND BIOLOGICAL SENSING APPLICATIONS}

In this section, other applications will be presented. It is important to distinguish two cases: those where the sensor is immersed in a liquid and those where it is not. The latter refers to a refractive index working point of 1 (air), whereas the former, with small variations depending on the type of liquid, refers to a working point of 1.33 . This is the reason why it is important to characterize the sensor as a function of the refractive index, as was shown in the previous section.

Regarding sensors working in air conditions, there are several possibilities. The traditional domains of application of optical sensors, before thin-film-based applications started, are temperature, strain, and pressure [69]. Temperature has only been studied theoretically with LMR-based sensors [70], because it is very difficult to overcome electronic temperature sensors, a technology that, without the need of a thin film, is simpler and effective. However, other 
applications are more challenging. The first one is the detection of gases. In 1995 it was possible to detect $\mathrm{n}$-heptane and iso-octane vapors with the polyimide thin film bexafluoro-isopropylidene-2,2'-bis[phthalic anhydride]-hexaituoro-iso-propylidene-2,2'-bis[4-amino benzene] (HFDA-HFDAM44), whereas the next year the same material was used for detection of $14 \%-$ $20 \%$ methanol atmosphere, 15\%-30\% ethanol atmosphere, and 30\%-60\% isopropanol atmosphere [11,12]. Later on, in 2010, ammonia with a $\mathrm{ZnO}$ thin film deposited on a D-shaped fiber was detected [28]. In addition to this, in 2012 it was possible to detect, with a thin film including an organic-metallic compound with chemical structure $\left[\mathrm{Au}_{2} \mathrm{Ag}_{2}\left(\mathrm{C}_{6} \mathrm{~F}_{\mathrm{5}}\right)_{4}\left(\mathrm{NH}_{3}\right)_{2}\right]_{m}$, vapors from ethanol, methanol, and isopropanol, with sensitivities of $0.067,0.131$, and 0.074 $\mathrm{nm} / \mathrm{ppm}$, respectively [71]. In 2013 a better performance was obtained with another organometallic compound $\left[A u_{2} A g_{2}\left(C_{6} F_{5}\right)_{4}\left(C_{6} H_{5} C \equiv C C_{6} H_{5}\right)_{2}\right]_{n}$, which permits one to attain sensitivities $0.417,0520$, and $263 \mathrm{~nm} / \mathrm{ppm}$, respectively, for vapors from the same liquids [72] (see Fig. 6(a), the response to ethanol).

Humidity sensors are another type of detector that can be included in the group of sensors that work at refractive index 1. Except for a thin film of water that is created on the surface of the thin film, the surrounding medium is air. The first study, published in 1988, was based on polyimide PI2566 (2,2'bis(3,4-dicarboxyphenyl) hexafluoroisopropylidene dianhydride + 4,4' oxydianiline), where intensity fluctuations were induced when changing the atmosphere humidity [10].

Two more studies were published in 2010 [22,25]. It is important to indicate that, contrary to sensors for the detection of gases, a double coating was deposited on a CRMF structure. The first layer of ITO was used to generate the LMR, whereas the second layer was a highly hydrophilic material (a hydrogel), which under changing humidity conditions experienced a variation in its properties. This caused a wavelength shift of the LMR. One of the materials used for the second thin film was agarose, which permitted one to obtain a sensitivity of $0.75 \mathrm{~nm}$ per percentage of relative humidity (nm/RH\%) [25]. The other article used the same structure, but the second thin film was a polymer (PAH/PAA) deposited with the layer-by-layer (LbL) technique [22]. One of the sensors had 20 bilayers deposited and the other one 100 bilayers. The sensor with the higher number of bilayers (higher thickness) showed a higher sensitivity of $5.4 \mathrm{~nm} / \mathrm{RH} \%$ (see Fig. 6(b)) compared to the $1.2 \mathrm{~nm} / \mathrm{RH} \%$ obtained for the sensor with a lower number of bilayers. However, 
the advantage of the latter sensor was that, due to its lower thickness, the response time was reduced. In a latter study, the performance of two relative humidity sensors working at the first and the second LMR was also analyzed. As expected, the most sensitive device was the sensor where the first LMR was tracked [48]. Finally, some interesting studies have been published on the possibility of embedding silver nanoparticles in a PAH/PAA matrix. This permits one to obtain both LMRs and the localized surface plasmon resonance (LSPR) [73,74]. The highest sensitivity attained was $1 \mathrm{~nm} / \mathrm{RH} \%$; human breath can also be tracked due to the fast response to humidity-less than 0.5 seconds [73].

Another interesting application is the detection of voltage. Again, a multilayer strategy was used. A high sensitivity of $0.4 \mathrm{~nm} / \mathrm{V}$ was attained by using a multilayer coating composed of a PVdF layer embedded in two ITO layers acting as electrodes [75] (see Fig. 6(c)). The first ITO layer generated the LMR and the external voltage induced a modification of the PVdF layer refractive index, which induced a wavelength shift of the LMR. This device can be used as a tunable optical filter of for sensing in high voltage lines.

With respect to sensors working in liquid, the first $\mathrm{pH}$ sensor was obtained with a mutilayer PAH/PAA thin film [26]. The principle in operation is that PAH and PAA are two weak polyelectrolytes whose degree of ionization changes as a function of $\mathrm{pH}$. Consequently, the bilayer thickness changes from very thin bilayers, if both polymers are fully ionized, to thick bilayers, if the degree of ionization of one of the polymers is reduced [76]. Since the central wavelength of the LMR depends on the coating thickness [17,42], there is a wavelength shift as a function of $\mathrm{pH}$. A sensitivity of $17.5 \mathrm{~nm}$ per $\mathrm{pH}$ unit is attained in the $\mathrm{pH}$ range 5-7 [26]. In an additional study, the sensitivity of two sensors coated with 25 or 100 bilayers of PAH/PAA was analyzed. A higher sensitivity was obtained with a lower coating thickness $(37 \mathrm{~nm}$ per $\mathrm{pH}$ unit, see Fig. 6d) compared to that reached with a higher coating thickness ( $25 \mathrm{~nm}$ per $\mathrm{pH}$ unit) [47]. This is because the first LMR was tracked for 25 bilayers, whereas the second resonance was monitored for 100 bilayers. Actually, the difference should be higher, but there is another phenomenon that counteracted: the swelling and deswelling of the PAH/PAA structure was higher as the thickness of this film increased. Finally, another sensor with PAH/PAA coating but with a single mode-tapered optical fiber was successfully proved [50]. The reduction of the LMR spectral width allowed one to distinguish, for specific $\mathrm{pH}$ values, the $\mathrm{LMR}_{\mathrm{TE}}$ and the $\mathrm{LMR}_{\mathrm{TM}}$ bands due to the two mode transitions that occurred. 
Regarding the time response of the sensors, those devices with lower number of bilayers, 25 or 30 , presented time responses of 30 seconds and 60 seconds, respectively [26,47,50], whereas for those with 100 bilayers, more than six hours of recovery time was required in some cases [41]. The possibility of detecting the concentration of chemical species has been successfully proved [34], when a $\mathrm{ZnO}$ coating was set on a CRMF, leading to the generation of LMRs that allowed the detection of $\mathrm{H}_{2} \mathrm{~S}$. In that work, the results were compared with those obtained with an SPR induced with combination of a silver coating and $\mathrm{ZnO}$. The main conclusion is that the LMR phenomenon overcame the SPR in this analysis: $0.65 \mathrm{~nm} / \mathrm{ppm}$ was obtained with an LMR whereas $0.2 \mathrm{~nm} / \mathrm{ppm}$ was obtained with an SPR. In another study, sensitivity to hydrogen gas $\left(\mathrm{H}_{2}\right)$ was studied with three different types of coatings: a single ITO thin film, a single ITO nanoparticle-based layer, and an ITO thin film plus an ITO nanoparticle layer. The best option was the last one, which improved the other two configurations by a factor of 3 and 1.5 [77].

The path to biosensors has also been opened with the detection of immunoglobulin $\mathrm{G}^{28}$. More recently it has been possible to detect antigliadin antibodies, an indicator of the presence of celiac disease, in a concentration of 5 ppm [52] (see Fig. 6(f)), which is lower than the range of medical diagnosis (8-10 ppm) [78].

Finally, another interesting sensing technology, aptasensors, has been proved successful for thrombin detection [79] and sepsis diagnosis [80]. 


\section{(a) Organic vapors}

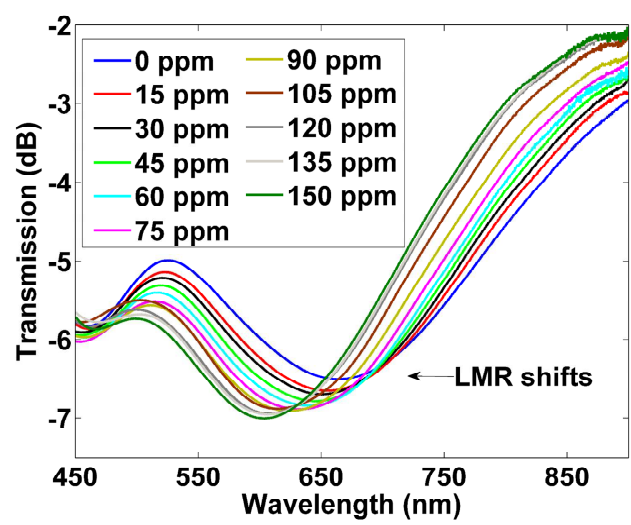

\section{(b) Relative humidity (RH)}

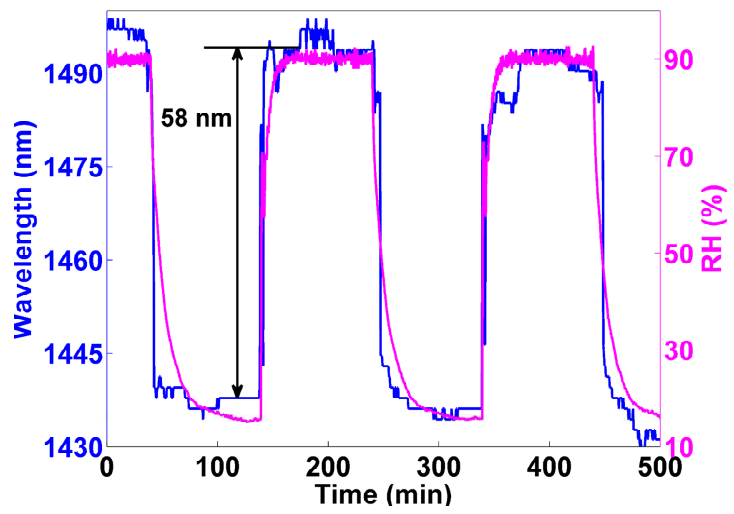

(c) Voltage
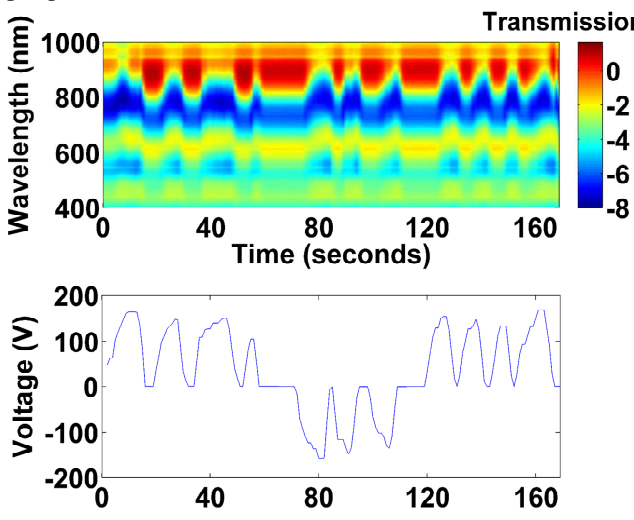

(d) $\mathrm{pH}$ of a solution

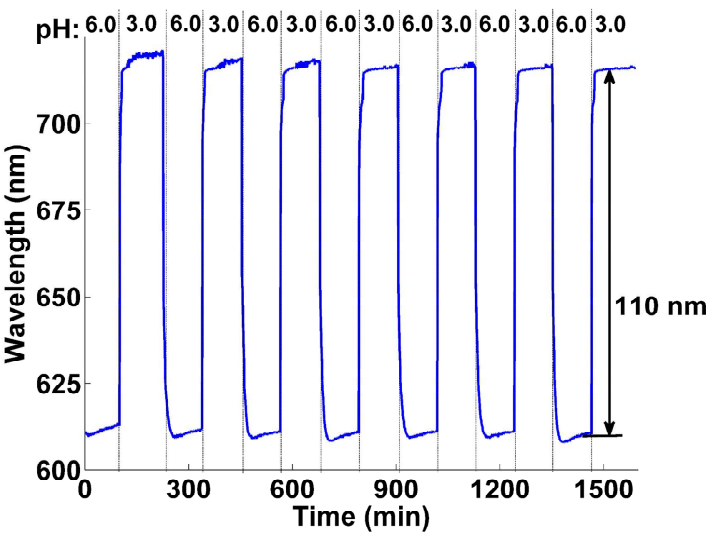

(e) Chemical sensors

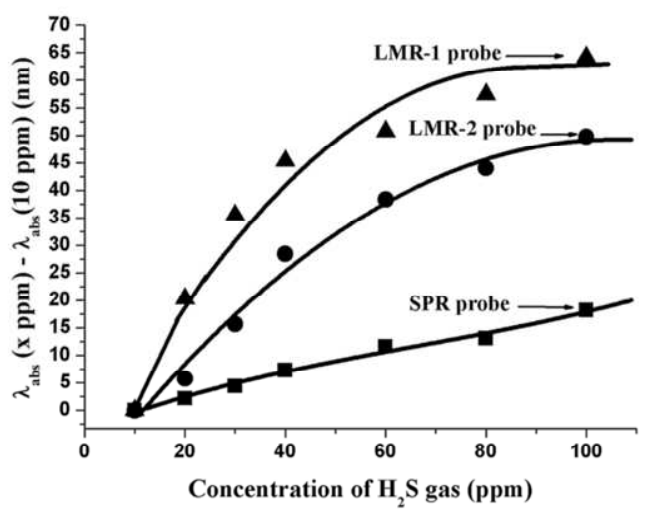

(f) Inmunosensors

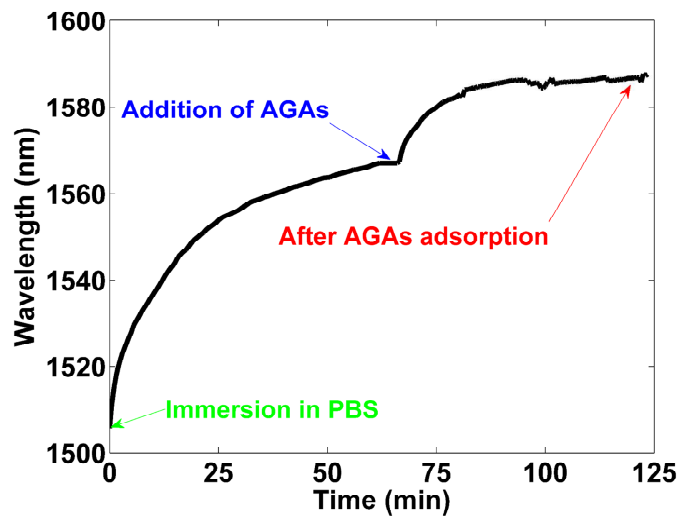

Fig. 6. (a) Detection of organic vapors. (b) Humidity sensor. (c) Voltage detection. (d) PH sensor. (e) $\mathrm{H}_{2} \mathrm{~S}$ sensor. (f) Immunosensor. Figure reproduced with permission from: a, ref. [72], ㄷ 2013 Elsevier; b, ref. [22], (C) 2010 Elsevier; c, ref. [75], (C) 2013 OSA; d, ref. [47], (C) 2011 Elsevier; e, ref. [34], (C) 2011 Elsevier; f, ref. [52], () 2014 Elsevier. 


\section{CONCLUSIONS AND OUTLOOK TO THE FUTURE}

Lossy-mode resonance-based sensors is still a young research field, but it must be underlined that during the last five years more than 50 studies have been published in different journals. This indicates that it could become a competitor of other traditional optical sensing platforms such as SPRs, long-period fiber gratings, or microring resonators.

We have discussed the principle in operation and the main characteristics of these devices. A lossy mode is guided in a thin film deposited on an optical structure, and a reorganization of the modes guided in the substrate takes place. Consequently, the imaginary part of the substrate modes increases and hence the propagation losses increase. This leads to the generation of an attenuation band in the optical spectrum.

LMRs can be excited with both TE and TM polarized light, and multiple resonance peaks can be obtained in the optical spectrum. Moreover, the position of the LMR in the optical spectrum can be tuned by varying the coating thickness.

The design rules for optimized devices were established for devices coated with a single thin film: increasing the refractive index and thickness of the thin film and the surrounding medium refractive index (SRMI), provided the first LMR is tracked. Since the SRMI is typically limited by the application and since the thickness is used to control the position of the LMR in the optical spectrum, the best choice is to increase the thin-film refractive index. So far, the best results with cladding- removed multimode fiber have been obtained with $\mathrm{SnO}_{2}$ coatings. However, there are new designs with two layers that permit increasing the sensitivity of single thin-film-based devices. This opens new paths to the optimization of the sensitivity of these devices.

Different optical structures have also been compared, and it has been proved that the selection of the structure permits one to reduce the LMR spectral width, which is interesting in order to increase the sensor resolution. The tapered single mode fiber configuration permits one to improve the performance, but the best choice so far is the D-shaped fiber.

Currently, the most active research field in LMRs consists of refractometer characterization, because obtaining a good refractometer is the basis for development of the subsequent chemical and biological sensors. However, LMR-based devices are not limited to these fields. They can be used for gas, humidity, and voltage sensing. Even aptasensors based on LMRs have been designed successfully. In view of the success obtained by LMR-based devices in such a short 
period and in important domains, such as the biosensors market, the LMR platform, with its continuous sensitivity improvement during recent years, is an ideal candidate platform for the development of the sensors of the future.

\section{ACKNOWLEDGEMENTS}

This work was supported in part by the Spanish Ministry of Education and Science-FEDER TEC2013-43679-R

\section{REFERENCES}

[1] D.K. Gramotnev, S.I. Bozhevolnyi, Plasmonics beyond the diffraction limit, Nat. Photonics. 4 (2010) 83 - 91.

[2] A. Koenderink, A.F., Alù, A., Polman, Nanophotonics: Shrinking light-based technology, Science 348 (2015) 516-521.

[3] T. Nylander, C. Liedberg, B. Lind, Gas detection by means of surface plasmon resonance, Sensors and Actuators. 3 (1982) 79-88.

[4] H. Kretschmann, E. Raether, Radiative decay of nonradiative surface plasmons excited by light, Zeitschrift Für Naturforsch. A. 23 (1968) 2135-2136.

[5] I. Liedberg, Bo Nylander, Claes Lunström, Surface plasmon resonance for gas detection and biosensing, Sensors and Actuators. 4 (1983) 299-304.

[6] J. Homola, Surface plasmon resonance sensors for detection of chemical and biological species, Chem. Rev. 108 (2008) 462-493. doi:10.1021/cr068107d.

[7] O.S. Wolfbeis, Fiber-Optic Chemical Sensors and Biosensors, Anal. Chem. 80 (2008) 4269-4283.

[8] M. Piliarik, J. Homola, Surface plasmon resonance (SPR) sensors: approaching their limits?, Opt. Express. 17 (2009) 16505-16517. doi:10.1364/OE.17.016505.

[9] Batchman T.E. McWright Glen M., Mode Coupling Between Dielectric and semiconductor planar waveguides, IEEE J. Quantum Electron. QE-18 (1982) 782-788.

[10] R. Reuter, H. Franke, Monitoring humidity by polyimide lightguides, Appl. Phys. Lett. 52 (1988) 778-779. doi:10.1063/1.99281.

[11] R.P. Podgorsek, H. Franke, Optical detection of water / alcohol vapours by polyimide lightguides, Opt. Detect. Water/alcohol Vapours by Polyim. Light. 30 (1996) 201-205. 
[12] R.P. Podgorsek, H. Franke, Selective optical detection of $\mathrm{n}$-heptane $\overline{1}$ so-octane vapors by polyimide lightguides, Opt. Detect. Water/alcohol Vapours by Polyim. Light. 20 (1995) 201-205.

[13] M. Marciniak, J. Grzegorzewski, M. Szustakowski, Analysis of lossy mode cut-off conditions in planar waveguides with semiconductor guiding layer, IEE Proc. J Optoelectron. 140 (1993) 247-252. doi:10.1049/ip-j.1993.0040.

[14] A. Andreev, B. Pantchev, P. Danesh, B. Zafirova, E. Karakoleva, E. Vlaikova, E. Alipieva, A refractometric sensor using index-sensitive mode resonance between singlemode fiber and thin film amorphous silicon waveguide, Sensors Actuators B Chem. 106 (2005) 484-488. doi:10.1016/j.snb.2004.09.002.

[15] I. Del Villar, V. Torres, M. Beruete, Experimental demonstration of lossy mode and surface plasmon resonance generation with Kretschmann configuration, Opt. Lett. 40 (2015) 4739-4382. doi:10.1364/OL.40.004739.

[16] V. Torres, M. Beruete, P. Sánchez, I. Del Villar, Indium tin oxide refractometer in the visible and near infrared via lossy mode and surface plasmon resonances with Kretschmann configuration, Appl. Phys. Lett. 108 (2016) 043507. doi:10.1063/1.4941077.

[17] I. Del Villar, C.R. Zamarreño, M. Hernaez, F.J. Arregui, I.R. Matias, Lossy mode resonance generation with indium-tin-oxide-coated optical fibers for sensing applications, J. Light. Technol. 28 (2010) 111-117.

[18] I. Del Villar, C.R. Zamarreño, P. Sanchez, M. Hernaez, C.F. Valdivielso, F.J. Arregui, I.R. Matias, Generation of lossy mode resonances by deposition of high-refractive-index coatings on uncladded multimode optical fibers, J. Opt. 12 (2010) 095503. doi:10.1088/2040-8978/12/9/095503.

[19] M. Hernaez, I. Del Villar, C.R. Zamarreño, F.J. Arregui, I.R. Matias, Optical fiber refractometers based on lossy mode resonances supported by TiO2 coatings, Appl. Opt. 49 (2010) 3980-3985.

[20] C.R. Zamarreño, M. Hernáez, I. Del Villar, I.R. Matías, S. Member, F.J. Arregui, ITO coated optical fiber refractometers based on resonances in the infrared region, IEEE Sens. J. 10 (2010) 365-366.

[21] C.R. Zamarreño, S. Lopez, M. Hernaez, I. Del Villar, I.R. Matias, F.J. Arregui, Resonance-based optical fiber refractometers, IEEE Photonics Technol. Lett. 22 (2010) 
1778-1780. doi:10.1109/ICSENS.2011.6127171.

[22] C.R. Zamarreño, M. Hernaez, I. Del Villar, I.R. Matias, F.J. Arregui, Tunable humidity sensor based on ITO-coated optical fiber, Sensors Actuators, B Chem. 146 (2010) 414417. doi:10.1016/j.snb.2010.02.029.

[23] C.R. Zamarreno, I. DelVillar, M. Hernaez, I.R. Matias, F.J. Arregui, Optical Fiber Refractometers with Tunable Sensitivity Based on Indium Tin Oxide Coatings, Sens Lett. 8 (2010) 744-746. doi:10.1166/s1.2010.1340.

[24] I. Del Villar, C.R. Zamarreño, M. Hernaez, F.J. Arregui, I.R. Matias, Resonances in coated long period fiber gratings and cladding removed multimode optical fibers: a comparative study., Opt. Express. 18 (2010) 20183-20189.

[25] M. Hernaez, C.R. Zamarreño, C. Fernandez-Valdivielso, I. Del Villar, F.J. Arregui, I.R. Matias, Agarose optical fibre humidity sensor based on electromagnetic resonance in the infra-red region, Phys. Status Solidi Curr. Top. Solid State Phys. 7 (2010) 2767-2769. doi:10.1002/pssc.200983815.

[26] C.R. Zamarreño, M. Hernáez, I. Del Villar, C. Fernandez-Valdivielso, F.J. Arregui, I.R. Matias, Optical fiber $\mathrm{pH}$ sensor fabrication by means of indium tin oxide coated optical fiber refractometers, Phys. Status Solidi Curr. Top. Solid State Phys. 7 (2010) 2705-2707. doi:10.1002/pssc.200983800.

[27] A.T. Andreev, B.S. Zafirova, E.I. Karakoleva, A.O. Dikovska, P.A. Atanasov, Highly sensitive refractometers based on a side-polished single-mode fibre coupled with a metal oxide thin-film planar waveguide, J. Opt. A Pure Appl. Opt. 10 (2008) 035303. doi:10.1088/1464-4258/10/3/035303.

[28] A.O. Dikovska, G.B. Atanasova, N.N. Nedyalkov, P.K. Stefanov, P.A. Atanasov, E.I. Karakoleva, A.T. Andreev, Optical sensing of ammonia using $\mathrm{ZnO}$ nanostructure grown on a side-polished optical-fiber, Sensors Actuators, B Chem. 146 (2010) 331-336. doi:10.1016/j.snb.2010.02.018.

[29] J. Hu, C.R. Menyuk, Understanding leaky modes: slab waveguide revisited, Adv. Opt. Photonics. 1 (2009) 58. doi:10.1364/AOP.1.000058.

[30] F. Yang, J.R. Sambles, Determination of the optical permittivity and thickness of absorbing films using long range modes, J. Mod. Opt. 44 (1997) 1155-1164. doi:10.1080/095003497153437. 
[31] D. Razansky, P.D. Einziger, D.R. Adam, Broadband absorption spectroscopy via excitation of lossy resonance modes in thin films, Phys. Rev. Lett. 95 (2005) 1-4. doi:10.1103/PhysRevLett.95.018101.

[32] N. Paliwal, J. John, Theoretical modeling of lossy mode resonance based refractive index sensors with ITO / TiO2 bilayers, Appl. Opt. 53 (2014) 3241-3246.

[33] D. Kaur, V.K. Sharma, a. Kapoor, High sensitivity lossy mode resonance sensors, Sensors Actuators, B Chem. 198 (2014) 366-376. doi:10.1016/j.snb.2014.03.058.

[34] S.P. Usha, S.K. Mishra, B.D. Gupta, Fiber optic hydrogen sulfide gas sensors utilizing $\mathrm{ZnO}$ thin film/ZnO nanoparticles: A comparison of surface plasmon resonance and lossy mode resonance, Sensors Actuators B Chem. 218 (2015) 196-204. doi:10.1016/j.snb.2015.04.108.

[35] C.R. Zamarreño, M. Hernaez, I.R. Matias, F.J. Arregui, Optical fiber sensors based on indium tin oxide surface plasmon resonance supporting coatings, in: Proc. SPIE 7503, 20th Int. Conf. Opt. Fibre Sensors, 2009: p. 75030W.

[36] M. Rani, N.K. Sharma, V. Sajal, Surface plasmon resonance based fiber optic sensor utilizing indium oxide, Optik (Stuttg). 124 (2013) 5034-5038. doi:10.1016/j.ijleo.2013.03.036.

[37] S.K. Mishra, S. Rani, B.D. Gupta, Surface plasmon resonance based fiber optic hydrogen sulphide gas sensor utilizing nickel oxide doped ITO thin film, Sensors Actuators, B Chem. 195 (2014) 215-222. doi:10.1016/j.snb.2014.01.045.

[38] I.H. Malitson, Interspecimen Comparison of the Refractive Index of Fused Silica, J. Opt. Soc. Am. 55 (1965) 1205-1209. doi:10.1364/JOSA.55.001205.

[39] A.B. Socorro, Study and design of thin-film-deposited optical biosensing devices based on wavelength detection of resonances PhD dissertation, Public University of Navarra, 2015.

[40] S. Lopez, I. Del Villar, C. Ruiz Zamarreño, M. Hernaez, F.J. Arregui, I.R. Matias, Optical fiber refractometers based on indium tin oxide coatings fabricated by sputtering, Opt. Lett. 37 (2012) 28-30. doi:10.1364/OL.37.000028.

[41] C.R. Zamarreño, S. Lopez, M. Hernaez, I. Del Villar, I.R. Matias, F.J. Arregui, Resonance-based refractometric response of cladding-removed optical fibers with sputtered indium tin oxide coatings, Sensors Actuators, B Chem. 175 (2012) 106-110. doi:10.1016/j.snb.2011.12.082. 
[42] I. Del Villar, M. Hernaez, C.R. Zamarreño, P. Sánchez, C. Fernández-Valdivielso, F.J. Arregui, I.R. Matias, Design rules for lossy mode resonance based sensors, Appl. Opt. 51 (2012) 4298.

[43] C.R. Zamarreno, P. Sanchez, M. Hernaez, I. Del Villar, C. Fernandez-Valdivielso, I.R. Matias, F.J. Arregui, Sensing Properties of Indium Oxide Coated Optical Fiber Devices Based on Lossy Mode Resonances, IEEE Sens. J. 12 (2012) 151-155. doi:10.1109/JSEN.2011.2142181.

[44] P. Zubiate, C.R. Zamarreño, I. Del Villar, I.R. Matias, F.J. Arregui, High sensitive refractometers based on lossy mode resonances (LMRs) supported by ITO coated Dshaped optical fibers, Opt. Express. 23 (2015) 8045-8050. doi:10.1364/OE.23.008045.

[45] C.R. Zamarreño, P. Zubiate, M. Sagües, I.R. Matias, F.J. Arregui, Experimental demonstration of lossy mode resonance generation for transverse-magnetic and transverse-electric polarizations, Opt. Lett. 38 (2013) 2481-2483. doi:10.1364/OL.38.002481.

[46] P. Zubiate, C.R. Zamarreño, I. Del Villar, I.R. Matias, S. Member, F.J. Arregui, Experimental Study and Sensing Applications of Polarization-Dependent Lossy Mode Resonances Generated by D-Shape Coated Optical Fibers, IEEE J. Light. Technol. 33 (2015) 2412-2418.

[47] C.R. Zamarreño, M. Hernáez, I. Del Villar, I.R. Matías, F.J. Arregui, Optical fiber pH sensor based on lossy-mode resonances by means of thin polymeric coatings, Sensors Actuators, B Chem. 155 (2011) 290-297. doi:10.1016/j.snb.2010.12.037.

[48] P. Sanchez, C.R. Zamarreno, M. Hernaez, I. Del Villar, I.R. Matias, F.J. Arregui, Considerations for lossy-mode resonance-based optical fiber sensor, IEEE Sens. J. 13 (2013) 1167-1171. doi:10.1109/JSEN.2012.2227717.

[49] A.B. Socorro, I. Del Villar, J.M. Corres, F.J. Arregui, I.R. Matias, Influence of waist length in lossy mode resonances generated with coated tapered single-mode optical fibers, IEEE Photonics Technol. Lett. 23 (2011) 1579-1581. doi:10.1109/LPT.2011.2164520.

[50] A.B. Socorro, I. Del Villar, J.M. Corres, F.J. Arregui, I.R. Matias, Tapered single-mode optical fiber $\mathrm{pH}$ sensor based on lossy mode resonances generated by a polymeric thinfilm, IEEE Sens. J. 12 (2012) 2598-2603.

[51] A.B. Socorro, J.M. Corres, I. Del Villar, F.J. Arregui, I.R. Matias, Fiber-optic biosensor 
based on lossy mode resonances, Sensors Actuators, B Chem. 174 (2012) 263-269. doi:10.1016/j.snb.2012.07.039.

[52] A.B. Socorro, I. Del Villar, J.M. Corres, F.J. Arregui, I.R. Matias, Spectral width reduction in lossy mode resonance-based sensors by means of tapered optical fibre structures, Sensors Actuators B Chem. 200 (2014) 53-60. doi:10.1016/j.snb.2014.04.017.

[53] C. Rhodes, S. Franzen, J.P. Maria, M. Losego, D.N. Leonard, B. Laughlin, G. Duscher, S. Weibel, Surface plasmon resonance in conducting metal oxides, J. Appl. Phys. 100 (2006) 54905. doi:10.1063/1.2222070.

[54] J. Homola, Surface Plasmon Resonance Based Sensors, Springer, 2006.

[55] I. Del Villar, C.R. Zamarreño, M. Hernaez, F.J. Arregui, I.R. Matias, Generation of lossy mode resonances with absorbing thin-films, J. Light. Technol. 28 (2010) 3351-3357.

[56] I. Del Villar, C.R. Zamarreño, M. Hernaez, P. Sanchez, F.J. Arregui, I.R. Matias, Generation of Surface Plasmon Resonance and Lossy Mode Resonance by thermal treatment of ITO thin-films, Opt. Laser Technol. 69 (2015) 1-7. doi:10.1016/j.optlastec.2014.12.012.

[57] J.M. Corres, I. Del Villar, F.J. Arregui, I.R. Matias, Analysis of lossy mode resonances on thin-film coated cladding removed plastic fiber, Opt. Lett. 40 (2015) 4867-4870. doi:10.1364/OL.40.004867.

[58] I. Del Villar, A.B. Socorro, M. Hernaez, J.M. Corres, C.R. Zamarreño, P. Sanchez, F.J. Arregui, I.R. Matias, Sensors Based on Thin-Film Coated Cladding Removed Multimode Optical Fiber and Single-Mode Multimode Single-Mode Fiber : A Comparative Study, J. Sensors. 763762 (2015) 1-7.

[59] A. Shalabney, I. Abdulhalim, Figure-of-merit enhancement of surface plasmon resonance sensors in the spectral interrogation, Opt. Lett. 37 (2012) 1175-1177. doi:10.1364/OL.37.001175.

[60] I. Del Villar, F.J. Arregui, J.M. Corres, C. Bariain, J. Goicoechea, C.R. Zamarreño, C. Elosua, M. Hernaez, P.J. Rivero, A.B. Socorro, A. Urrutia, P. Sanchez, P. Zubiate, D. Lopez, N. De Acha, J. Ascorbe, I.R. Matias, Nanocoated Optical Fibre for Lossy Mode Resonance ( LMR ) Sensors and Filters, in: IEEE Int. Conf. Transparent Opt. Networks, 2015: p. 7193695.

[61] P. Sanchez, C.R. Zamarreño, M. Hernaez, I.R. Matias, F.J. Arregui, Optical fiber 
refractometers based on Lossy Mode Resonances by means of SnO2 sputtered coatings, Sensors Actuators, B Chem. 202 (2014) 154-159. doi:10.1016/j.snb.2014.05.065.

[62] M. Zourob, S. Mohr, P.R. Fielden, N.J. Goddard, Small-volume refractive index and fluorescence sensor for micro total analytical system ( $\mu$-TAS) applications, Sensors Actuators, B Chem. 94 (2003) 304-312. doi:10.1016/S0925-4005(03)00460-X.

[63] S. Zhu, F. Pang, S. Huang, F. Zou, Y. Dong, T. Wang, High sensitivity refractive index sensor based on adiabatic tapered optical fiber deposited with nanofilm by ALD, Opt. Express. 23 (2015) 13880-13888. doi:10.1364/OE.23.013880.

[64] R. Slavík, J. Homola, Ultrahigh resolution long range surface plasmon-based sensor, Sensors Actuators, B Chem. 123 (2007) 10-12. doi:10.1016/j.snb.2006.08.020.

[65] F.J. Arregui, I. Del Villar, J.M. Corres, J. Goicoechea, C.R. Zamarreño, C. Elosua, M. Hernaez, P.J. Rivero, A.B. Socorro, A. Urrutia, P. Sanchez, P. Zubiate, D. Lopez, N. De Acha, I.R. Matias, Fiber-optic Lossy Mode Resonance Sensors, in: Procedia Eng., Elsevier B.V., 2014: pp. 3-8. doi:10.1016/j.proeng.2014.11.253.

[66] F.J. Arregui, I. Del Villar, C.R. Zamarreño, P. Zubiate, I.R. Matias, Giant Sensitivity of Optical Fiber Sensors by means of Lossy Mode Resonance, Sensors Actuators B Chem. 232 (2016) 660-665. doi:10.1016/j.snb.2016.04.015.

[67] M. Smietana, M.L. Korwin-Pawlowski, W.J. Bock, G.R. Pickrell, J. Szmidt, Refractive index sensing of fiber optic long-period grating structures coated with a plasma deposited diamond-like carbon thin film, Meas. Sci. Technol. 19 (2008) 085301. doi:10.1088/09570233/19/8/085301.

[68] R. Horvath, L.R. Lindvold, N.B. Larsen, Reverse-symmetry waveguides: Theory and fabrication, Appl. Phys. B Lasers Opt. 74 (2002) 383-393. doi:10.1007/s003400200823.

[69] B. Lee, Review of the present status of optical fiber sensors, Opt. Fiber Technol. 9 (2003) 57-79. doi:10.1016/S1068-5200(02)00527-8.

[70] N. Paliwal, J. John, Theoretical modelling of lossy mode resonance (LMR) based fiber optic temperature sensor utilizing TiO 2 sensing layer, in: 12th Int. Conf. Fiber Opt. Photonics, 2014: p. M4A.22.

[71] C. Elosua, F.J. Arregui, C.R. Zamarreño, C. Bariain, A. Luquin, M. Laguna, I.R. Matias, Volatile organic compounds optical fiber sensor based on lossy mode resonances, Sensors Actuators B Chem. 173 (2012) 523-529. doi:10.1016/j.snb.2012.07.048. 
[72] C. Elosúa, I. Vidondo, F.J. Arregui, C. Bariain, a. Luquin, M. Laguna, I.R. Matías, Lossy mode resonance optical fiber sensor to detect organic vapors, Sensors Actuators, B Chem. 187 (2013) 65-71. doi:10.1016/j.snb.2012.09.046.

[73] P.J. Rivero, A. Urrutia, J. Goicoechea, F.J. Arregui, Optical fiber humidity sensors based on Localized Surface Plasmon Resonance (LSPR) and Lossy-mode resonance (LMR) in overlays loaded with silver nanoparticles, Sensors Actuators, B Chem. 173 (2012) 244249. doi:10.1016/j.snb.2012.07.010.

[74] P.J. Rivero, a. Urrutia, J. Goicoechea, I.R. Matias, F.J. Arregui, A Lossy Mode Resonance optical sensor using silver nanoparticles-loaded films for monitoring human breathing, Sensors Actuators, B Chem. 187 (2013) 40-44. doi:10.1016/j.snb.2012.09.022.

[75] J.M. Corres, J. Ascorbe, F.J. Arregui, I.R. Matias, Tunable electro-optic wavelength filter based on lossy-guided mode resonances, Opt. Express. 21 (2013) 31668-31677. doi:10.1364/OE.21.031668.

[76] J. Choi, M.F. Rubner, Influence of the degree of ionization on weak polyelectrolyte multilayer assembly, Macromolecules. 38 (2005) 116-124. doi:10.1021/ma048596o.

[77] S.K. Mishra, S.P. Usha, B.D. Gupta, A lossy mode resonance-based fiber optic hydrogen gas sensor for room temperature using coatings of ITO thin film and nanoparticles, Meas. Sci. Technol. 27 (2016) 045103. doi:10.1088/0957-0233/27/4/045103.

[78] C. Catassi, E. Fabiani, M. Gasparin, R. Troncone, Quantitative antigliadin anti- body measurement in clinical practice: an Italian multicentre study, Ital. J. Gastroenterol. Hepatol. 31 (1999) 366-370.

[79] L. Razquin, C.R. Zamarreno, F.J. Munoz, I.R. Matias, F.J. Arregui, Thrombin detection by means of an aptamer based sensitive coating fabricated onto LMR-based optical fiber refractometer, in: Proc. IEEE Sensors, 2012: p. 6411186. doi:10.1109/ICSENS.2012.6411186.

[80] C.R. Zamarreño, I. Ardaiz, L. Ruete, F.J. Muñoz, I.R. Matias, F.J. Arregui, C-reactive protein aptasensor for early sepsis diagnosis by means of an optical fiber device, IEEE SENSORS 2013 - Proc. (2013) 4-7. doi:10.1109/ICSENS.2013.6688222. 